Methods We systematically reviewed English-language studies reporting on CT screening programmes in educational settings (school/college/university) published between 2005 and 2011. We classified programmes into groups on the basis of screening strategies and report the median testing rate (number invited/screened) and CT positivity from studies where data were available.

Results We identified 28 studies describing 32 screening programmes in America/Canada $(n=13)$, Europe $(n=8)$, Australia/ New Zealand $(n=7)$ and Asia $(n=4)$. Most targeted both male and female students (71\%). Programs were in secondary schools $(n=14)$, post-secondary schools $(\mathrm{n}=16)$ and both secondary and post-secondary schools $(n=2)$. Across all programmes, 55369 tests were conducted. The highest testing rates were in programmes involving screening students in class rooms (four programmes), opportunistic screening of students undergoing routine health examinations (six programmes), and opportunistic screening of students visiting school-based health centres for other reasons (six programmes), with median testing rates of $66 \%, 54 \%$ and $46 \%$ respectively. Lower testing rates were found in programmes involving screening in other school locations e.g. canteen/study stall (four programmes) with a median testing rate of $30 \%$. The median CT positivity was 4.7\% (range: $1.3-18.1 \%$ ); $4.1 \%$ in males, $5.8 \%$ females.

Conclusion The review demonstrated that education facilities can be used for CT screening. Testing programmes were established in a range of educational facilities, in a variety of countries, and accessed large numbers of males and females. The CT positivity supports educational institutions as a setting to conduct screening. Targeting students in classrooms and opportunistic screening at school clinics and routine health examinations appears to achieve high testing rates in the school setting.

\section{P3.367 SERO-PREVALENCE OF SEXUALLY TRANSMITTED DISEASE (HIV, SYPHILIS, HEPATITIS-B AND HEPATITIS-C) IN VOLUNTEER DONORS OF GAOL INMATES AND STUDENT COMMUNITY IN PUNJAB PROVINCE OF PAKISTAN}

doi:10.1136/sextrans-2013-051184.0820

M I Oadeer, S Hasnain, H Yasmeen. Department of Microbiology and Molecular Genetics University of the Punjab Lahore, Lahore, Pakistan

Objective Cohort studies of prisoner and student community volunteer blood donors recruited in 2007-2012 in 30 gaols and 30 educational institues of Punjab province. In Punjab, there are 32 prisoners gaol that are nearly three times overcrowded with 62500 prisoners (undertrial, convict and condemned prisoners). A number of studies indicate that even in prisons of developed countries prevalence of transmission of sexually transmitted infections and HIV is high.

Methods Ten thousand-5000 each fromjail and educational institutes) apparently healthy donors were assessed for the sero-prevalence of human immunodeficiency virus (HIV), hepatitis B virus (HBV), hepatitis C virus (HCV) and syphillus (RPR) using a commercially available Enzyme Linked Immunosorbent Assay (ELISA)based kit. Information was obtained for risk factors using structured questionnaire.

Results Out of the 5,000 samples screened in each community, 337 $(6.74 \%)$ in student community and $1424(28.48 \%)$ in prisoner community were sero-positive. Subjects aged $15-45$ years recorded $2.20 \%$ HBV, 4.12\% HCV, $0.42 \%$ RPR and no HIV positivity in students while in prisons $5.28 \% \mathrm{HBV}, 12.32 \% \mathrm{HCV}, 0.18 \% \mathrm{HIV}$ and $10.70 \%$ RPR positivity was recorded. Subjects aged 15-25 years are more HBV positive $(2.51 \%)$ and $(7.94 \%)$ while subjects aged $25-35$ years were more HCV positive $(4.88 \%)$ and $(14.18 \%)$ in student and prisoner community respectively. Unfortunately, sero-prevalnce rate is high in prisoner community as compared to student community.

Conclusion Overcrowding, poor hygienic and close living conditions stake prisoners at a very high risk for acquisition of sexually transmitted infections as compare to student community. Public awareness and vaccination programme should be improved in the community on urgent basis.

\section{P3.368 STD CLINIC PATIENTS' PREFERENCES FOR HIV PREVENTION STRATEGIES}

doi:10.1136/sextrans-2013-051184.0821

J G Castro, D Jones, I Granowsky, B Alshehry, S Weiss. University of Miami, Miami, $F L$, United States

Background More information is needed to understand how the newer HIV prevention methods should be positioned and which mix of prevention methods should be offered and promoted within the at risk populations. This study sought to obtain data about the preferences for effective biomedical interventions by individuals from the diverse ethnic and racial backgrounds that comprise the STD clinic populations in Miami

Methods A cross sectional survey was used to assess knowledge and preference of traditional (condoms) and new biomedical methods to prevent HIV (Circumcision-C-, Pre-exposure prophylaxis -PreP and microbicides $-M$ ) in STD clinic patients. After an initial assessment, the study coordinator provided basic simple descriptions of three new methods of HIV prevention by pamphlets and/or recorded video. The relative preference for each of all the prevention strategies was re-assessed information was provided.

Results Thirty five participants are reported in this interim analysis; 55\% were female; 58\% were African American; 25\% were Hispanic and $12 \%$ were Haitians. Most of the participants were not aware of the efficacy of C (68\%), PreP (77\%) or M $(79 \%)$ in decreasing the risk of acquiring HIV infection. At baseline, participants described as their preferred method to prevent HIV the use of male condoms (77\%) and had marginal preference for the newer methods $C(3 \%), M(6 \%)$ and PreP (3\%). After the information about the new methods was provided, most of the participants reported to be aware of these methods (80\%) and although male condoms was still the first choice for most of the participants (46\%) a higher percentage of participants preferred M $(20 \%)$ and PreP (14\%).

Conclusions STD clinic patients who participated in this study had very limited knowledge about the new biomedical strategies to prevent HIV infection. A brief informational session can increase their willingness to use the newer HIV preventive strategies.

\section{P3.369 ACCEPTABILITY OF HPV TETRAVALENT VACCINE AMONG MALES ATTENDING THE STD CLINIC OF MILAN - THE IMPORTANCE OF THE COSTS FOR PATIENTS}

doi:10.1136/sextrans-2013-051184.0822

M Cusini, S Ramoni. Fondazione IRCCS Ca' Granda Ospedale Maggiore Policlinico Department of Dermatology, Milan, Italy

Background HPV infection is usually transmitted by sexual contact and represent the most prevalent sexually transmitted infection all over the world. The clinical spectrum of HPV infection varies from the asymptomatic status to benign genital lesions to the development of virus associated tumours. There is no direct antiviral drug for HPV and all available therapies are aimed to the destruction of lesions or to the enhancement of the immune response.

In recent year a vaccine for prevention of infection has been developed and used for the vaccination of young adolescent girls and later of young women. The quadrivalent vaccine directed against HPV types 6, 11, 16 and 18 has shown efficacy on the incidence of genital warts not only in the vaccinated population but also in the male population of comparable age. Further researches 